\title{
Multi-wavelength follow-up observations of ANTARES neutrino alerts
}

\author{
Damien Dornic* \\ CPPM, Aix-Marseille Université, CNRS/IN2P3, 13009 Marseille, France. \\ E-mail: dornicecppm.in2p3.fr \\ D. Turpin (IRAP), M. Ageron, V. Bertin, J. Brunner, A. Mathieu (CPPM), F. Schüssler, \\ B. Vallage (CEA/IRFU), S. Basa (LAM) on behalf the ANTARES, TAROT, MASTER, \\ ZADKO, Swift, MWA, H.E.S.S. Collaborations.
}

\begin{abstract}
High-energy neutrinos could be produced in the interaction of charged cosmic rays with matter or radiation surrounding astrophysical sources. Transient phenomena, such as gamma-ray bursts, core-collapse supernovae or active galactic nuclei are promising candidates to emit high-energy neutrinos. To search for coincidences between a transient event and a neutrino emission, a followup program of neutrino alerts is in operation within the ANTARES Collaboration since 2009. This program triggers a network of robotic optical telescopes immediately after the detection of a neutrino event and schedule several observations in the following weeks. The most interesting neutrino candidates are also followed by the Swift XRT telescope and the Murchison Wide field Array radio telescope and the H.E.S.S. very high-energy gamma-ray telescope. By combining the information provided by the ANTARES neutrino telescope with information coming from other observatories, the probability of detecting a source is enhanced, allowing the possibility of identifying a neutrino progenitor from a single detected event. No significant counterpart associated with a neutrino emission has been identified during image analysis.
\end{abstract}

35th International Cosmic Ray Conference - ICRC2017

10-20 July, 2017

Bexco, Busan, Korea

${ }^{*}$ Speaker. 


\section{Introduction}

High-energy neutrinos may be produced in the interaction of charged cosmic rays with matter or radiation surrounding astrophysical sources. Even with the recent detection of extraterrestrial high-energy neutrinos by the IceCube experiment [1,2], no astrophysical neutrino source has yet been discovered. Such a detection would provide a direct evidence of hadronic acceleration mechanisms and would therefore solve the origin of very high-energy cosmic rays.

High-energy neutrinos are thought to be produced in several kinds of astrophysical sources, such as the galactic binaries, supernova remnant, gamma-ray busts (GRB) [3], core-collapse supernovae (CCSN) [4] or active galactic nuclei (AGN) [5]. Most of these sources are also transient events covering a large range in the time domain, from seconds for GRB to weeks for CCSN or AGN. By combining the information provided by the ANTARES neutrino telescope [6] with information coming from other observatories, the probability of detecting a source is enhanced since the neutrino background is significantly reduced in the time window around the event [7].

Based on this idea, a multi-wavelength follow-up program of ANTARES alerts, dubbed TAToO (Telescopes-Antares Target of Opportunity), has been operating since 2009 [8]. It triggers multiwavelength observations within a few seconds after the detection of selected high-energy neutrino candidates. The document is organized as follow: Section 2 describes the alert sending system and its performances. Follow-up observations of neutrino candidates are now performed over a broad range of the electromagnetic spectrum, from visible to X-ray. Section 3 describes the multiwavelength facilities used by ANTARES to follow the neutrino alerts. Section 4 reports the main results of the visible and X-ray observations. Section 5 shows the results of the multi-wavelength and multi-messenger follow-up of the alert ANT150901A. The recent search for radio counterpart of few high-energy neutrino events by the Murchison Widefield Array (MWA) [9] is reported in Section 6. On the extreme opposite of the electromagnetic band, a few ANTARES alerts have been followed looking for $\mathrm{TeV}$ photons detected by H.E.S.S. [10] (Section 7).

\section{Characteristics of the ANTARES alert sending system}

All the ANTARES events are reconstructed in real-time $(<5 \mathrm{~s})$ by two independent algorithms $[11,12]$. These algorithms use an idealized detector geometry and are independent of the dynamical positioning calibration (the positions of the optical modules are not corrected for displacements due to sea currents). This reconstruction and subsequent quality selections allow the rate of events to be reduced from few $\mathrm{Hz}$ down to few $\mathrm{mHz}$ removing almost all the huge downgoing atmospheric muon contribution. The duty cycle of TAToO is around $90 \%$. From the remaining atmospheric neutrino sample, the selection of the neutrino candidates with an increased probability to be of cosmic origin is performed with three online neutrino trigger criteria currently implemented in the TAToO alert system [8]:

- High energy trigger: the detection of a single high energy neutrino.

- Directional: the detection of a single neutrino for which the direction points toward $\left(<0.4^{\circ}\right)$ a local galaxy $(<20 \mathrm{Mpc})$ in the GWGC catalogue [13]. 
Table 1: Performances of the three alert criteria. The third column shows the median angular resolution at the mean energy $<\mathrm{E}>$. The fourth column corresponds to the fraction of events inside a $2^{\circ} \times 2^{\circ}$ field of view assuming a flux of GRB [3] and CCSN [4].

\begin{tabular}{ccccc}
\hline \hline Trigger & $<\mathrm{E}\rangle$ & Ang. Res. & Fraction & Muon contamination \\
\hline Doublet & $\sim 100 \mathrm{GeV}$ & $\leq 0.7^{\circ}$ & $/$ & $0 \%$ \\
\hline Single HE & $\sim 7 \mathrm{TeV}$ & $\sim 0.3^{\circ}$ & $96 \%(\mathrm{GRB}) 68 \%(\mathrm{SN})$ & $<0.1 \%$ \\
\hline Single directional & $\sim 1 \mathrm{TeV}$ & $\sim 0.4^{\circ}$ & $90 \%(\mathrm{GRB}) 50 \%(\mathrm{SN})$ & $\sim 2 \%$ \\
\hline
\end{tabular}

- Doublet trigger: the detection of at least two neutrinos coming from similar directions $\left(<3^{\circ}\right)$ within a predefined time window (15 min).

The main performances of these three triggers are described in Table 1. Until now, no doublet trigger has been sent to the network. The trigger criteria are inspired by the features expected from astrophysical sources and are tuned to comply with the alert rate to send to the telescope network. An agreement between ANTARES and the optical telescope collaborations allows a rate of around 25 alerts per year to be sent to each optical telescope, while an agreement to send 6 alerts per year to the Swift satellite have been accepted. Due to this reduced rate, a subset of the high-energy trigger with more restrictive requirements on the neutrino energy, provides a dedicated trigger for the Swift satellite. A similar sub-sample is sent to the H.E.S.S. and M.W.A telescopes. The TAToO alert system is able to send alerts within few seconds (3-5 s) after the neutrino detection with an angular resolution better than $\sim 0.4^{\circ}$.

\section{Multi-wavelength follow-up facilities}

The ANTARES neutrino alerts are followed by the small robotic optical telescopes such as TAROT [14], ZADKO [15], MASTER [16] and SVOM/GWAC [17] located all-around the world. TAROT is a network of two identical $0.25 \mathrm{~m}$ telescopes with a field of view (FoV) of $\sim 1.86^{\circ} \times$ $1.86^{\circ}$ located in Calern (France) and La Silla (Chile). Zadko is a 1 meter telescope located at the Gingin observatory in Western Australia. As its FoV is about 0.15 square degrees, seven tiles are needed to cover the ANTARES point spread function. The 6 MASTER telescopes are located in Russia, Canary Islands and in South Africa, and consist of 6 pairs of tubes with a diameter of 0.40 m covering a FoV of up to 8 square degrees for each pair of telescopes. Until the end of 2014, the network also comprises the four optical telescopes ROTSE [18], which have progressively stopped their activity. These telescopes reach a limiting magnitude of $~ 19-20.5$ mag depending on their diameters. In 2017, the follow-up has been extended to the SVOM/GWAC telescopes located in China providing a very large $\mathrm{FoV}\left(\sim 40^{\circ}\right)$ but with a not very deep sensitivity ( $\sim 15$ mag). The wide FoV and the fast response of these telescopes (images can be taken less than $20 \mathrm{~s}$ after the neutrino detection) are well suited to the search for transient sources. For each alert, the optical observation strategy is composed of an early follow-up (within 24 hours after the neutrino detection), to search for fast transient sources such as GRB afterglows, complemented by several observations during the two following months, to detect for example the rising light curves of CCSN. Each observation is composed of series of optical images (with clear filter). Optical images are analyzed with dedicated pipelines based on the image subtraction method to look for new transient sources [22]. 
The Swift satellite with its XRT [19] provides a unique opportunity to observe X-ray counterparts to neutrino triggers. The detection sensitivity of the XRT is $\sim 5 \times 10^{-13} \mathrm{erg} \mathrm{cm}^{-2} \mathrm{~s}^{-1}$ in $1 \mathrm{ks}$ exposure, and an energy band from 0.3 to $10 \mathrm{keV}$ is covered [20]. Due to the small FoV of the XRT (radius of $\sim 0.2^{\circ}$ ) and the typical error radius of an ANTARES alert $\left(\sim 0.3-0.4^{\circ}\right)$, each observation of a neutrino trigger is composed of 4 tiles of $2 \mathrm{ks}$ each. This mapping covers about $72 \%$ of the ANTARES point spread function for a high-energy neutrino. The observation strategy is composed of an automatic response to the neutrino trigger with observations starting as soon as possible. There is an online analysis of the data and in the case where an interesting candidate to be the counterpart is found, further observations are scheduled.

In the last few years, follow-up observations of a sub-sample of neutrino alerts are also performed by the Murchinson WideField Array (MWA [9]) which is the low frequency (80 - 300 $\mathrm{MHz}$ ) precursor of the Square Kilometre Array. Its huge field of view (700 square degrees at 150 $\mathrm{MHz}$ ) is particularly valuable for follow-up of neutrino candidates, which have rather large position uncertainties. A few alerts have also triggered observations by the H.E.S.S. Cherenkov telescope located in Namibia. H.E.S.S. has a typical energy threshold of $100 \mathrm{GeV}$ and a large field of view of around $5^{\circ}[10]$.

\section{Optical and X-ray follow-up}

Since mid 2009, a total of 256 alerts have been sent to optical robotic telescopes while 13 targets of opportunity have been sent to the XRT instrument on board the Swift satellite since mid2013. The typical follow-up efficiency is around $80 \%$ for the network of robotic telescopes and for the Swift satellite.

From mid 2009 to June 2017, 169/256 alerts with early optical follow-up ( $<24 \mathrm{~h}$ after the neutrino time) have been analyzed ( $\sim 66 \%$ of the sent alerts). Among them, 40 have a delay lower than 1 min $(\sim 16 \%)$. No optical counterparts were found and upper limits on the R-band magnitude of a transient astrophysical source have been derived. These limits correspond to the limiting magnitude of images, which is the faintest signal that can be detected. As we are looking for rapidly-fading sources, the signal is supposed to be more important in the first image of the observation, so the upper limits are the limiting magnitude of each first image computed at $5 \sigma$ and corrected for Galactic extinction [13]. By comparing these upper limits with optical afterglow light curves of gamma-ray bursts (Figure 1), it becomes possible to reject a GRB association with each neutrino alert, in particular when the optical follow-up is performed within a few minutes after the neutrino trigger [22]. A similar analysis has been carried out with Swift-XRT follow-ups of 13 ANTARES alerts [22]. The typical delay of the first Swift observation is around $6 \mathrm{~h}$ with a minimum delay of $1.1 \mathrm{~h}$. The probability to reject the GRB hypothesis reaches more than $\sim 70 \%$ if the X-ray follow-up occurs within few hours after the trigger.

Among the 256 sent alerts, 177 have sufficiently good optical long-term follow-ups, i.e. at least 3 (2) nights of observation for TAROT (MASTER) network. No significant slowly varying transient optical counterparts were found in association with a neutrino trigger. The expected number of accidental SN detections, i.e. a SN detection in coincidence with a background neutrino event, is estimated to 0.3 in the 177 alerts assuming a rate of SN of 1 per year within a sphere of $10 \mathrm{Mpc}$ 
$\left(2.4 \times 10^{-4} \mathrm{yr}^{-1} \mathrm{Mpc}^{-3}\right)$. This result is consistent with the small expectation SN number with a probability of 0.74 .

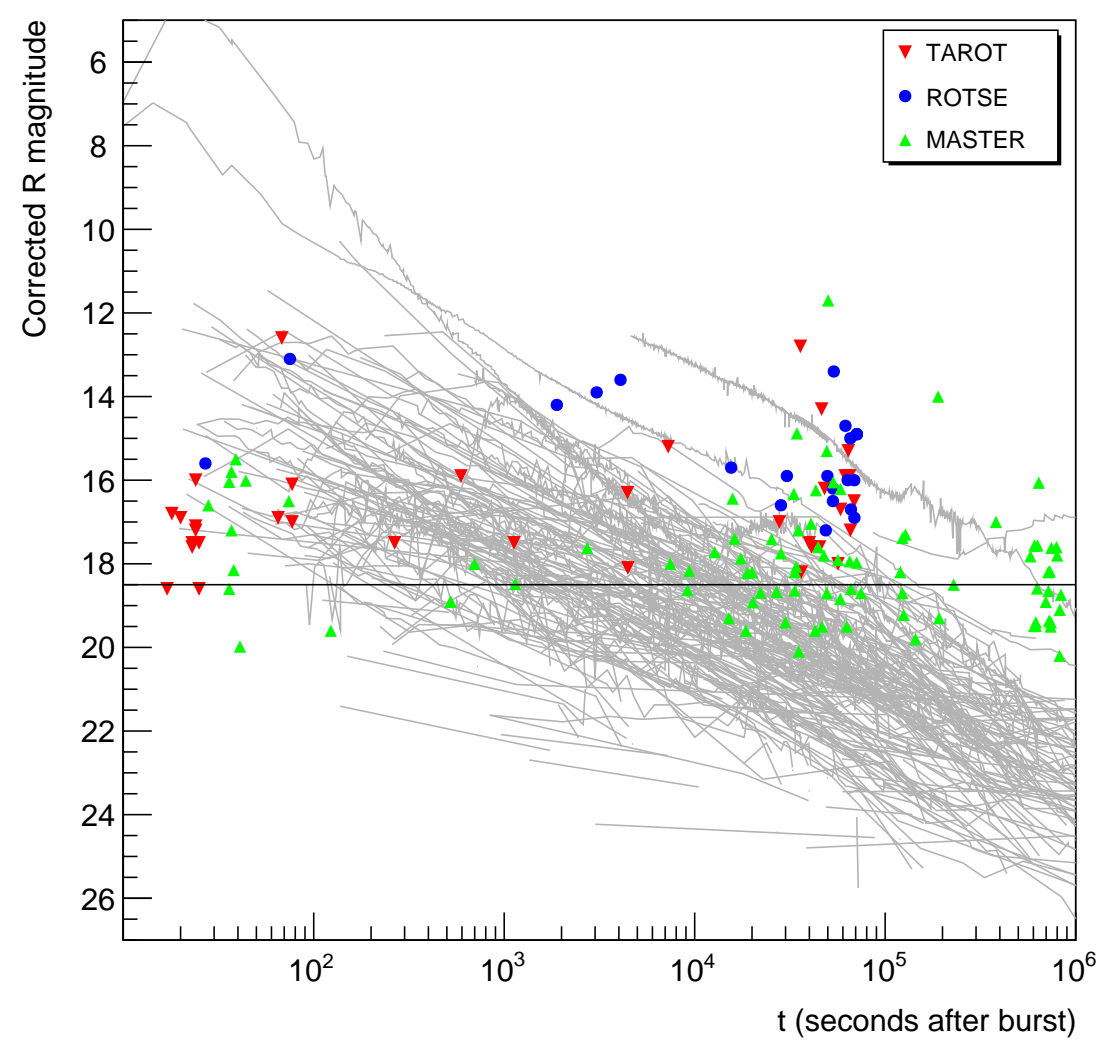

Figure 1: Comparison between archived optical light-curves for 301 GRBs detected in the period 19972014 and the upper limits obtained for the 169 alerts. Red, blue and green markers indicate upper limits on GRB afterglow magnitudes for neutrino alerts observed by TAROT, ROTSE and MASTER respectively. The horizontal black line corresponds to the typical sensitivity of the optical telescopes.

\section{Follow-up of ANT150901A}

On September 2015 1st, ANTARES detected a bright neutrino (ANT150901A) with an energy around $50 \mathrm{TeV}$. The direction is $\mathrm{RA}=16 \mathrm{~h} 25^{\prime} 42$, DEC $=-27 \mathrm{~d} 23^{\prime} 24$ with an uncertainty of $\sim 18$ arcmin (radius, 50\% containment). An alert has been sent to our follow-up partners after a delay of 10 s. Swift/XRT has started the follow-up 9h after the neutrino trigger. 8 sources have been identified in the field of view, among them 5 are catalogued, 2 faint sources and one uncatalogued, relatively bright and variable X-ray source above the Rosat All-Sky Survey (RASS) limit [23] $\left(=5 \times 10^{-13}\right.$ $-1.4 \times 10^{-12} \mathrm{erg} \mathrm{cm}^{-2} \mathrm{~s}^{-1}$ at $\left.0.3-10 \mathrm{keV}\right)$. This source is located at $0.14^{\circ}$ from the neutrino direction. Further observations with XRT show an outburst with a typical lenght of around 2 days (Figure 2 left). In parallele, MASTER has followed the field of view after 10h. No significant new 
variable source has been identified. At the position of the X-ray source, MASTER has identified a bright star (USNO-B1.0 0626-0501169) of a magnitude 12.3 with a light curve showing no flux and no color variations. The Figure 2 (right) illustrates the field of view of MASTER.

A GCN notice [24] and an ATeL telegram [25] were emitted to request for more multiwavelenght observations to characterize the star and to test the association between the X-ray flare and this bright star. 19 multi-wavelength observatories have answered to this trigger covering the full electro-magnetic spectrum: 1 radio telescope, 11 optical/IR telescopes, $4 \mathrm{X}$-ray satellites and 4 very high-energy gamma-ray observatories. IceCube has also reported a non-observation. These observations point to USNO-B1.0 0626-0501169 being a binary RS CVn system, undergoing a flaring episode that produced the X-ray emission. The typical characteristics of this star are a distance of around $100-150 \mathrm{pc}$, an age of around $10 \mathrm{Myr}$ and a mass around $0.75 \mathrm{M}_{\odot}$. Therefore, this source seems not to be the origin of the bright ANT150901A neutrino with a probability of $\sim 3 \%$ of chance association.
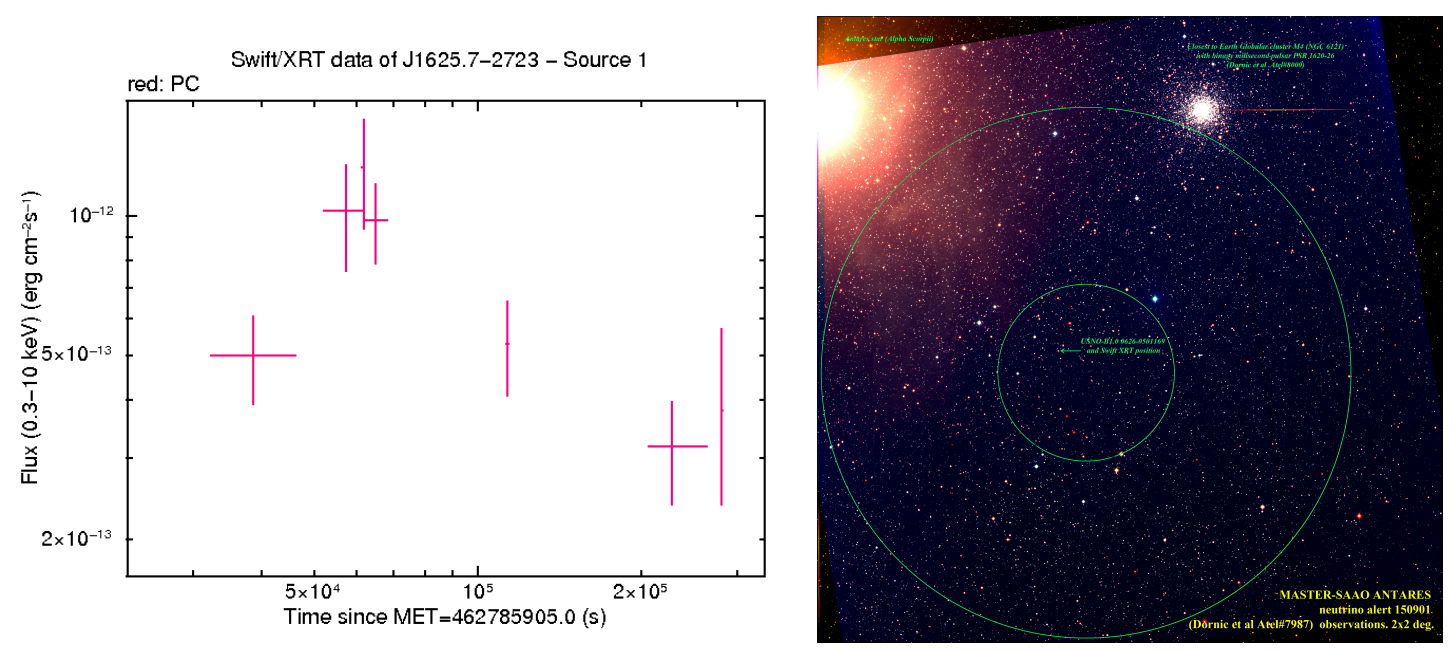

Figure 2: Left: Light curve mesured by the XRT on board of Swift for the X-ray source identified in the follow-up of ANT150901A. Right: Field of view of MASTER corresponding to ANT150901A.

\section{Radio follow-up with MWA}

A search for radio counterparts to two candidate high-energy neutrino events detected in November 2013 (ANT131121A) and March 2014 (ANT140323A) was performed using the Murchison Widefield Array [26]. Such triggers have directions consistent $\left(<0.4^{\circ}\right)$ with the positions of galaxies within $20 \mathrm{Mpc}$ of Earth [13]. Two galaxies match in each case: NGC1374 and ESO358015 match ANT131121A, and ESO499-037 and PGC29194 match ANT140323A. PGC29194 (the Antlia Dwarf Galaxy), at a distance of $1.3 \mathrm{Mpc}$, is located just 6' from the neutrino position. Both neutrino events also had optical follow-up. For ANT131121A, 12 observations of 6 images were performed with the TAROT telescope in Chile from 2 - 61 days after the trigger. No optical tansient was identified, to a limiting magnitude of 19. For ANT140323A, a total of 8 images were taken with ROTSE $3 \mathrm{~b}$ in Texas (starting $\sim 15 \mathrm{hr}$ after the trigger) and 10 images with TAROT Chile up to 
45 days after the trigger according to the long-term strategy. No transient counterpart was found to limiting magnitudes of 16.4 (prompt) and 18.7 (long-term).

Transient or strongly variable radio sources consistent with the neutrino positions are looked for using MWA archival data at frequencies between 118 and $182 \mathrm{MHz}$, taken 20 days prior to, at the same time as, and up to a year after the neutrino triggers. As no such counterparts are detected, and $5 \sigma$ upper limit for low-frequency radio emission of $\sim 10^{37} \mathrm{erg} \mathrm{s}^{-1}$ for progenitors at $20 \mathrm{Mpc}$. If the neutrino sources are instead not in nearby galaxies, but originate in binary neutron star coalescences, the limits constrain the progenitors to be at $\mathrm{z} \geq 0.2$. ANTARES is now sending a sub-sample of the neutrino alerts in real-time to MWA. Analysis of MWA data is still on-going.

\section{Very high-energy gamma-ray follow-up with H.E.S.S.}

Since 2016, ANTARES and H.E.S.S. have signed an MoU to exchange information and alerts. Exploiting the intimate connection between high-energy neutrinos and very high-energy gamma rays, the very high gamma-ray H.E.S.S. telescopes have followed two ANTARES alerts shortly after the neutrino detection: ANT150901A and ANT170130A. For ANT150901, the observations started on September 3rd, 2015, at 18:58 UT as soon as the necessary observation conditions were reached. No very-high energy gamma-ray source has been identified in the $1.5 \mathrm{~h}$ observations. An upper limit on the gamma-ray flux to $\Phi(\mathrm{E}>320 \mathrm{GeV}$; 99\% C.L. $)<2.710^{-8} \mathrm{~m}^{-2} \mathrm{~s}^{-1}$ [27]. The neutrino ANT170130A direction has also been followed by H.E.S.S. with a very short delay, $32 \mathrm{~s}$ during 1 hour and again $45 \mathrm{~min}$ the night after. The preliminary analysis on site shows no source detection in the neutrino field of view [28] (detailed analysis are still in progress).

\section{Conclusion \& perspectives}

Follow-up of high-energy neutrino alerts is a very promising method to identify transient sources as sources of neutrino production. The detection of one counterpart associated in time and direction with one neutrino could laid to a high significance discovery. ANTARES is able to send alerts to the external community in 5-7 seconds after the time of the neutrino detection with a precision of the direction better than 0.4 degres. The triggers are followed by several multiwavelength facilities such as robotic telescopes located all around the world, a radio telescope, one $\mathrm{X}$-ray satellite and very high-energy gamma-ray telescopes. This provides an unique follow-up network covering the whole EM spectrum. Up to now, no counterpart has been detected significantly in the different searches and constraints have been set on the origin of individual neutrinos.

The two KM3NeT detectors [29], in construction in the Mediterranean Sea, will have a 50 times increased discovery potential compared to the ANTARES telescope from $5 \mathrm{GeV}$ to a few $\mathrm{PeV}$. Dedicated follow-up programs are in development which will allow to send more promising neutrino alerts to the astronomy community.

\section{References}

[1] M.G. Aartsen et al, Science, 342, 1, 2013.

[2] M.G. Aartsen et al, ApJ, 809(1), 98, 2015. 
[3] E. Waxman, J. Bahcall, Phys. Rev. Lett., 78, 2292-2295, 1997. P. Mészáros, E. Waxman, Phys. Rev. Lett., 87(17)171102, 2001. S. Hümmer, M.Rüger, F. Spanier, W. Winter, ApJ, 721, 630-652, 2010.

[4] S. Ando \& J.F. Beacom, Phys. Rev. Lett., 95 (6)061103, 2005.

[5] M. Böttcher, A. REimer, K. Sweeney, A. Prakash, ApJ, 768, 54, 2013.

[6] M. Ageron et al (ANTARES Collaboration), Nucl. Instr. Meth. A, 656, 11-38, 2011.

[7] S. Adrián-Martínez et al (ANTARES Collaboration), 2015, JCAP, 12, 014.

[8] M. Ageron et al (ANTARES Collaboration), Astrpart. Phys., 35, 530-536, 2012.

[9] C.J. Lonsdale, R.J. Capallo, M.F. Morales et al , 2009 IEEE proceedings, 97. 1497. S.J. Tingay, R. Gaeker, J.D. Bowman et al, 2013, PASA, 32, 25.

[10] F. Aharonian et al (H.E.S.S. Collaboration), A\&A 457 (2006) 899.

[11] J.A. Aguilar et al (ANTARES Collaboration), Astropart. Phys., 34, 652-662, 2011.

[12] S. Adrián-Martínez et al (ANTARES Collaboration), Astrophys. J. Lett., 743, 14-19, 2011.

[13] D.J. White, E.J. Daw, V.S. Dhillon, Classical and Quantum Gravity, 28(8):080516, 2011.

[14] A. Klotz, M; Boer, J.-L. Atteia, B. Gendre, AJ, 137, 4100-4108, 2009.

[15] D.M. Coward et al, 2016, PASA, 34, 5.

[16] V. Lipunov et al, Adv. in Astron., 30, 2010.

[17] Wei J., Cordier B et al (SVOM Collaboration), 2016, arXiv:1610.06892.

[18] C.W. Akerlof et ak, PASP, 115, 132-140, 2003.

[19] D.N. Burrows et al, Space Sci. Rev., 120, 165-195, 2005.

[20] P.A. Evans et al, ApJS, 210, 8, 2014.

[21] D.J. Schlegel, D.P. Finkbeiner, M. Dabvis, ApJ, 500, 525-553, 1998.

[22] S. Adrián-Martínez et al (ANTARES Collaboration), 2016, JCAP, 02, 062.

[23] W. Vogues et ak, A\&A, 349, 389-405, 1999.

[24] D. Dornic et al (ANTARES Collaboration), GCN, 18231 (2015).

[25] D. Dornic et al (ANTARES Collaboration), ATeL, 7987 (2015).

[26] S. Croft et al (MWA \& ANTARES Collaborations), The Astrophys. J. 823 (2016) no.2; L24.

[27] F. Schüssler, Proceedings of the Rencontres de Moriond 2017 (VHEPU), arXiv: 1705.08258.

[28] F. Schüssler, this conference.

[29] S. Adrián-Martínez et al, (KM3NET Collaboration), Journal of Physics G: Nuclear and Particle Physics, 43 (8), 084001, 2016. 